\title{
Kinetic equilibrium of a periodically twisted ellipse-shaped charged-particle beam
}

\author{
Jing Zhou and Chiping Chen \\ Intense Beam Theoretical Research Group, Plasma Science and Fusion Center, Massachusetts Institute of Technology, Cambridge, \\ Massachusetts 02139, USA
}

(Received 26 July 2006; published 26 October 2006)

\begin{abstract}
A Vlasov equilibrium of the Kapchinskij-Vladimirskij form is obtained for a periodically twisted ellipse-shaped charged-particle beam in a nonaxisymmetric periodic magnetic focusing field. The singleparticle Hamiltonian dynamics is analyzed self-consistently. A constant of motion analogous to the Courant-Snyder invariant is found. The equilibrium distribution function is constructed. The statistical properties of the beam equilibrium are studied. In the zero-temperature limit, the generalized envelope equations derived from the kinetic equilibrium theory recover the generalized envelope equations obtained in the cold-fluid equilibrium theory. Examples of periodically twisted elliptic beam equilibria are presented, and potential applications are discussed. For ribbon-beam amplifier and ribbon-beam klystron applications, the kinetic equilibrium theory predicts that the effect of beam temperature on the beam envelopes is negligibly small.
\end{abstract}

DOI: 10.1103/PhysRevSTAB.9.104201

PACS numbers: 29.27.-a, 52.59.Sa, 41.75.-i, 52.25.Dg

\section{INTRODUCTION}

A fundamental understanding of the kinetic equilibrium and stability properties of high-intensity electron and ion beams in periodic focusing fields is desirable in the design and operation of particle accelerators [1-14], such as storage rings and $\mathrm{rf}$ and induction linacs, as well as vacuum electron devices, such as klystrons and traveling-wave tubes with periodic permanent magnet (PPM) focusing. There are two well-known equilibria for periodically focused intense beams, including the KapchinskijVladmirskij (KV) equilibrium [7-9] in an alternatinggradient quadrupole magnetic focusing field and the periodically focused rigid-rotor Vlasov equilibrium $[10,11]$ in a periodic solenoid magnetic focusing field. In general, for linear focusing forces, self-consistent beam distributions can be formally constructed using a matrix formulation $[12,13]$.

It was shown formally in Ref. [12] that self-consistent beam distributions can be obtained that allow elliptical space-charge beams of arbitrary aspect ratio and with arbitrary rotation angle of the ellipse as long as the field is linear. However, obtaining concrete equilibria with nonupright ellipses is nontrivial. The previous explicitly known Vlasov equilibria of KV form [7,9-11] for highintensity, space-charge-dominated charged-particle beams propagating in the alternating-gradient quadrupole magnetic focusing field and the periodic solenoid magnetic focusing field charged-particle beams are circular on average; that is, the averages of the beam envelopes in different transverse directions over one period are the same.

There is considerable interest in the research and development of high-intensity charged-particle beams with a large aspect ratio transverse to the direction of propagation. First, large-aspect-ratio elliptic beams (or ribbon beams) can transport larger amounts of beam currents at reduced intrinsic space-charge forces and energies. Second, they couple efficiently to planar or rectangular rf structures. The combination of the space-charge reduction and efficient coupling allows efficient rf generation in vacuum electronic devices, and efficient acceleration in particle accelerators. Third, elliptic beams provide an additional adjustable parameter (e.g., the aspect ratio) which may be useful for better matching a beam into a periodic focusing channel [14].

One important application of ribbon beams is in the development of advanced radiation devices such as ribbon-beam amplifiers (RBAs) and ribbon-beam klystrons (RBKs), which have advantages over the corresponding conventional (circular-beam) devices in terms of efficiency and operational parameters. Other applications include the development of advanced accelerators capable of generating nonconventional beams, e.g., a planar radio-frequency (rf) linac producing ribbonlike bunches of charged particles.

Although sheet beams have been discussed in the literature for four decades, the Vlasov equilibrium of a highintensity, space-charge-dominated beam with a large-aspect-ratio elliptic cross section has not been discovered until this paper. Sturrock [15] first suggested use of a periodic magnetic focusing consisting of an array of planar-wiggler magnets for rectilinear beams. Zhang et al. [16] had some modest success in the experimental demonstration of the transport of a low-intensity (10 A, $500 \mathrm{kV}$ ) sheet beam in a planar-wiggler magnetic field, and observed considerable beam loss. Researchers made use of the multiple-time-scale analysis and the paraxial approximations to obtain the smooth-beam approximation of high-intensity sheet beam equilibria [17,18]. Recently, Russell et al. demonstrated the transformation of a circular beam into a sheet beam using asymmetric lenses [19]. The authors discovered a cold-fluid equilibrium for a high- 
intensity periodically twisted elliptic beam in a nonasymmetric periodic magnetic focusing field [6].

In this paper, it is shown that there exists a Vlasov equilibrium for a periodically twisted large-aspect-ratio intense charged-particle beam with uniform density in the transverse plane propagating through a nonaxisymmetric periodic magnetic focusing field. The single-particle Hamiltonian of such a periodically twisted large-aspectratio elliptic beam is investigated. The constant of motion analogous to the Courant-Snyder invariant [20] is found. The equilibrium beam distribution is constructed. The beam envelope equations and flow velocity equations are derived. In the zero-temperature limit, they are consistent with the generalized envelope equations derived from coldfluid equilibrium theory [6]. Statistical properties and possible applications of the present beam equilibrium are discussed.

\section{VLASOV EQUILIBRIUM THEORY}

We consider an ellipse-shaped, continuous, intense charged-particle beam propagating with constant axial velocity $\beta_{b} c \mathbf{e}_{z}$ through an applied nonaxisymmetric periodic magnetic focusing field. The applied nonaxisymmetric periodic magnetic focusing field inside the thin beam can be approximated by

$\mathbf{B}^{\mathrm{ext}} \cong-B_{0} \sin \left(k_{0} s\right) \mathbf{e}_{z}+B_{0} \cos \left(k_{0} s\right)\left[\frac{k_{0 x}^{2}}{k_{0}} x \mathbf{e}_{x}+\frac{k_{0 y}^{2}}{k_{0}} y \mathbf{e}_{y}\right]$,

where $s=z$ is the axial coordinate, $k_{0}=2 \pi / S, k_{0 x}^{2}+$ $k_{0 y}^{2}=k_{0}^{2}$, and $S$ is the axial periodicity length. The 3D magnetic field in Eq. (4) is fully specified by the three parameters $B_{0}, S$, and $k_{0 x} / k_{0 y}$. The associated magnetic vector potential can be expressed as

$$
\mathbf{A}^{\mathrm{ext}}=-B_{0} \sin \left(k_{0} s\right)\left[-\frac{k_{0 y}^{2}}{k_{0}^{2}} y \mathbf{e}_{x}+\frac{k_{0 x}^{2}}{k_{0}^{2}} x \mathbf{e}_{y}\right] .
$$

To determine the self-electric and self-magnetic fields of the beam self-consistently in the present paraxial approximation, we assume that the density profile of the beam is uniform inside the beam boundary, i.e.,

$$
n_{b}(x, y)=\frac{N_{b}}{\pi a(s) b(s)} \Theta\left(1-\frac{\tilde{x}^{2}}{a^{2}(s)}-\frac{\tilde{y}^{2}}{b^{2}(s)}\right) .
$$

In Eq. (3), $\quad \tilde{x}=x \cos [\theta(s)]+y \sin [\theta(s)]$ and $\tilde{y}=$ $-x \sin [\theta(s)]+y \cos [\theta(s)]$ represent the twisted coordinate as illustrated in Fig. 1; $\theta(s)$ is the twist angle of the ellipse; $\Theta(x)=1$ if $x>0$ and $\Theta(x)=0$ if $x<0$. The density of the elliptic beam with semimajor axis $a(s)=a(s+S)$ and semiminor axis $b(s)=b(s+S)$ is uniform in the beam interior $\left(\tilde{x}^{2} / a^{2}+\tilde{y}^{2} / b^{2} \leq 1\right)$. The semimajor and semiminor axes have the same periodicity as the

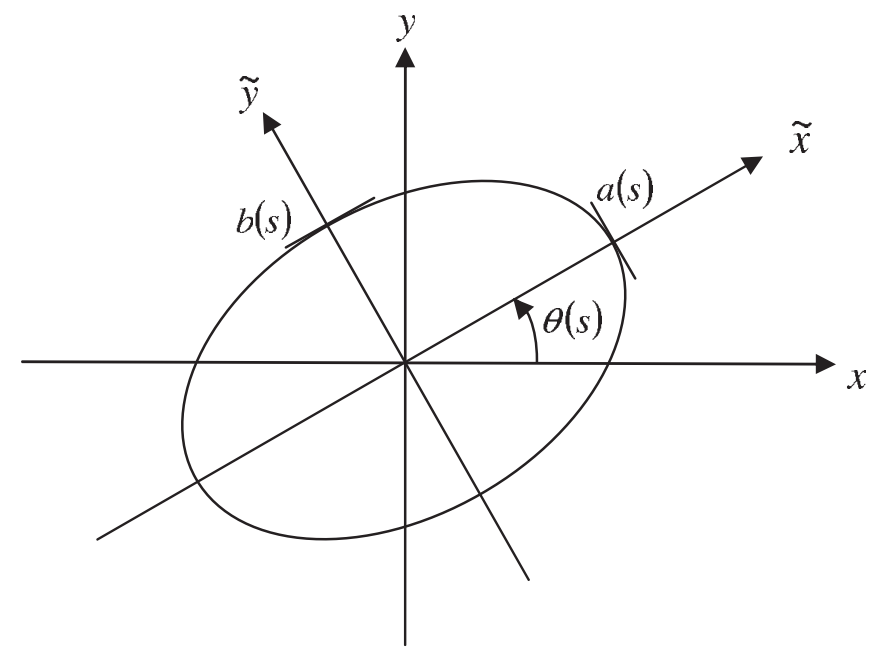

FIG. 1. Laboratory and twisted coordinate systems.

applied magnetic field with $S=2 \pi / k_{0} . \quad N_{b}=$ $2 \pi \int^{\infty} d x d y n_{b}(x, y, s)=$ const is the number of particles per unit axial length. In the paraxial approximation, the Budker parameter of the beam is assumed to be small, i.e., $q^{2} N_{b} / m c^{2} \ll \gamma_{b}$, and the transverse kinetic energy of a beam particle is assumed to be small compared with its axial kinetic energy. Here, $c$ is the speed of light in vacuo, $\gamma_{b}=\left(1-\beta_{b}^{2}\right)^{-1 / 2}$ is the relativistic mass factor, and $q$ and $m$ are the particle charge and rest mass, respectively.

From the equilibrium Maxwell equations, we find that the self-electric and self-magnetic fields, $\mathbf{E}^{s}$ and $\mathbf{B}^{s}$, are given by

$$
\begin{gathered}
\mathbf{E}^{s}(\tilde{x}, \tilde{y}, s)=\frac{2 q N_{b}}{a(s)+b(s)}\left[\frac{\tilde{x}}{a(s)} \mathbf{e}_{\tilde{x}}+\frac{\tilde{y}}{b(s)} \mathbf{e}_{\tilde{y}}\right], \\
\beta_{b}^{-1} \mathbf{B}^{s}(\tilde{x}, \tilde{y}, s)=\frac{2 q N_{b}}{a(s)+b(s)}\left[-\frac{\tilde{y}}{b(s)} \mathbf{e}_{\tilde{x}}+\frac{\tilde{x}}{a(s)} \mathbf{e}_{\tilde{y}}\right],
\end{gathered}
$$

in the beam interior $\left(\tilde{x}^{2} / a^{2}+\tilde{y}^{2} / b^{2} \leq 1\right)$. It is convenient to express the self-fields in terms of the scalar and vector potentials defined for $\tilde{x}^{2} / a^{2}+\tilde{y}^{2} / b^{2} \leq 1$ by

$$
\begin{aligned}
\Phi^{s}(\tilde{x}, \tilde{y}, s) & =\beta_{b}^{-1} A_{z}^{s}(\tilde{x}, \tilde{y}, s) \\
& =-\frac{2 q N_{b}}{a(s)+b(s)}\left[\frac{\tilde{x}^{2}}{a(s)}+\frac{\tilde{y}^{2}}{b(s)}\right]
\end{aligned}
$$

where $\quad \mathbf{A}^{s}(\tilde{x}, \tilde{y}, s)=A_{z}^{s}(\tilde{x}, \tilde{y}, s) \mathbf{e}_{z}, \quad \mathbf{E}^{s}(\tilde{x}, \tilde{y}, s)=$ $-\left(\mathbf{e}_{\tilde{x}} \partial / \partial \tilde{x}+\mathbf{e}_{\tilde{y}} \partial / \partial \tilde{y}\right) \Phi^{s}, \quad$ and $\quad \mathbf{B}^{s}=\left(-\mathbf{e}_{\tilde{y}} \partial / \partial \tilde{x}+\right.$ $\left.\mathbf{e}_{\tilde{x}} \partial / \partial \tilde{y}\right) A_{z}^{s}$.

In the paraxial approximation, the transverse motion for an individual particle in the combined self-fields and applied magnetic field is described by the normalized perpendicular Hamiltonian $\hat{H}_{\perp}=H_{\perp} / \gamma_{b} \beta_{b} m c^{2}$, 


$$
\begin{aligned}
\hat{H}_{\perp}\left(x, y, P_{x}, P_{y}, s\right)= & \frac{1}{2}\left[\left(P_{x}+\sqrt{\kappa_{z}(s)} \frac{k_{0 y}^{2}}{k_{0}^{2}} y\right)^{2}\right. \\
& \left.+\left(P_{y}-\sqrt{\kappa_{z}(s)} \frac{k_{0 x}^{2}}{k_{0}^{2}} x\right)^{2}\right] \\
& -\frac{K}{a+b}\left[\frac{\tilde{x}^{2}}{a}+\frac{\tilde{y}^{2}}{b}\right] .
\end{aligned}
$$

where $\left(x, P_{x}\right)$ and $\left(y, P_{y}\right)$ are canonical conjugate pairs, $\sqrt{\kappa_{z}(s)}=q B_{z}(s) / 2 \gamma_{b} \beta_{b} m c^{2}, \quad K=2 q^{2} N_{b} / \gamma_{b}^{3} \beta_{b}^{2} m c^{2} \quad$ is the self-field perveance, and the normalized transverse canonical momentum $\mathbf{P}_{\perp}=\left(P_{x}, P_{y}\right)$ is related to the transverse mechanical momentum $\mathbf{p}_{\perp}$ by $\mathbf{P}_{\perp}=\left(\gamma_{b} \beta_{b} m c\right)^{-1} \times$ $\left(\mathbf{p}_{\perp}+q \mathbf{A}_{\perp}^{\mathrm{ext}} / c\right)$.

It is convenient to transform the Hamiltonian in the Cartesian canonical coordinate $\left(x, y, P_{x}, P_{y}\right)$ to a new canonical coordinate $\left(x_{1}, y_{1}, P_{x 1}, P_{y 1}\right)$, so that the new Hamiltonian assumes a simpler form from which the invariants of motion are easily identified. The transformation of the Hamiltonian from the Cartesian canonical coordi- nate $\left(x, y, P_{x}, P_{y}\right)$ to the canonical coordinate $\left(x_{1}, y_{1}, P_{x 1}, P_{y 1}\right)$ involves two steps.

We first transform the Cartesian canonical coordinate $\left(x, y, P_{x}, P_{y}\right)$ to a twisted canonical coordinate $\left(\tilde{x}, \tilde{y}, \tilde{P}_{x}, \tilde{P}_{y}\right)$ using the generating function

$$
\begin{aligned}
F_{2}\left(x, y ; \tilde{P}_{x}, \tilde{P}_{y}, s\right)= & \tilde{P}_{x}[x \cos \theta(s)+y \sin \theta(s)] \\
& +\tilde{P}_{y}[-x \sin \theta(s)+y \cos \theta(s)] .
\end{aligned}
$$

It follows from Eq. (8) that

$$
\begin{aligned}
P_{x} & =\tilde{P}_{x} \cos \theta(s)-\tilde{P}_{y} \sin \theta(s), \\
P_{y} & =\tilde{P}_{x} \sin \theta(s)+\tilde{P}_{y} \cos \theta(s), \\
\tilde{x} & =x \cos \theta(s)+y \sin \theta(s), \\
\tilde{y} & =-x \sin \theta(s)+y \cos \theta(s) .
\end{aligned}
$$

The Hamiltonian in the twisted canonical coordinate is then expressed as

$$
\begin{aligned}
\tilde{H}_{\perp}\left(\tilde{x}, \tilde{y}, \tilde{P}_{x}, \tilde{P}_{y}, s\right)= & \hat{H}_{\perp}\left(x, y, P_{x}, P_{y}, s\right)+\partial F_{2} / \partial s \\
= & \frac{1}{2}\left[\tilde{P}_{x} \cos \theta(s)-\tilde{P}_{y} \sin \theta(s)+\sqrt{\kappa_{z}(s)} \frac{k_{0 y}^{2}}{k_{0}^{2}} \tilde{x} \sin \theta(s)+\sqrt{\kappa_{z}(s)} \frac{k_{0 y}^{2}}{k_{0}^{2}} \tilde{y} \cos \theta(s)\right]^{2} \\
& +\frac{1}{2}\left[\tilde{P}_{x} \sin \theta(s)+\tilde{P}_{y} \cos \theta(s)-\sqrt{\kappa_{z}(s)} \frac{k_{0 x}^{2}}{k_{0}^{2}} \tilde{x} \cos \theta(s)+\sqrt{\kappa_{z}(s)} \frac{k_{0 x}^{2}}{k_{0}^{2}} \tilde{y} \sin \theta(s)\right]^{2} \\
& -\frac{K}{a+b}\left(\frac{\tilde{x}^{2}}{a}+\frac{\tilde{y}^{2}}{b}\right)+\left(\tilde{P}_{x} \tilde{y}-\tilde{P}_{y} \tilde{x}\right) \frac{d \theta(s)}{d s}
\end{aligned}
$$

The equations of motion associated with the Hamiltonian in Eq. (10) are

$$
\begin{aligned}
\tilde{x}^{\prime}= & \frac{\partial \tilde{H}_{\perp}}{\partial \tilde{P}_{x}}=\tilde{P}_{x}+C(s) \tilde{x}+\left[\theta^{\prime}(s)-\alpha_{x}(s)\right] \tilde{y}, \\
\tilde{y}^{\prime}= & \frac{\partial \tilde{H}_{\perp}}{\partial \tilde{P}_{y}}=\tilde{P}_{y}-C(s) \tilde{y}-\left[\theta^{\prime}(s)-\alpha_{y}(s)\right] \tilde{x}, \\
\tilde{P}_{x}^{\prime}= & -\frac{\partial \tilde{H}_{\perp}}{\partial \tilde{x}}=-\left\{\kappa_{z}(s) \frac{k_{0 x}^{4} \cos ^{2}[\theta(s)]+k_{0 y}^{4} \sin ^{2}[\theta(s)]}{k_{0}^{4}}-\frac{2 K}{a(s)[a(s)+b(s)]\}}\right\} \tilde{x}-C(s) \tilde{P}_{x}+\left[\theta^{\prime}(s)-\alpha_{y}(s)\right] \tilde{P}_{y} \\
& -\kappa_{z}(s)\left(\frac{k_{0 y}^{4}-k_{0 x}^{4}}{k_{0}^{4}}\right) \frac{\sin [2 \theta(s)]}{2} \tilde{y}, \\
\tilde{P}_{y}^{\prime}= & -\frac{\partial \tilde{H}_{\perp}}{\partial \tilde{y}}=-\left\{\kappa_{z}(s) \frac{k_{0 x}^{4} \sin ^{2}[\theta(s)]+k_{0 y}^{4} \cos ^{2}[\theta(s)]}{k_{0}^{4}}-\frac{2 K}{b(s)[a(s)+b(s)]\}} \tilde{y}+C(s) \tilde{P}_{y}-\left[\theta^{\prime}(s)-\alpha_{x}(s)\right] \tilde{P}_{x}\right. \\
& -\kappa_{z}(s)\left(\frac{k_{0 y}^{4}-k_{0 x}^{4}}{k_{0}^{4}}\right) \frac{\sin [2 \theta(s)]}{2} \tilde{x},
\end{aligned}
$$

where prime denotes derivative with respect to $s$,

$$
C(s) \equiv \sqrt{\kappa_{z}(s)} \frac{k_{0 y}^{2}-k_{0 x}^{2}}{2 k_{0}^{2}} \sin [2 \theta(s)]
$$




$$
\alpha_{x}(s)=-\sqrt{\kappa_{z}(s)}\left\{\frac{k_{0 x}^{2}}{k_{0}^{2}} \sin ^{2}[\theta(s)]+\frac{k_{0 y}^{2}}{k_{0}^{2}} \cos ^{2}[\theta(s)]\right\},
$$

and

$$
\alpha_{y}(s)=-\sqrt{\kappa_{z}(s)}\left\{\frac{k_{0 x}^{2}}{k_{0}^{2}} \cos ^{2}[\theta(s)]+\frac{k_{0 y}^{2}}{k_{0}^{2}} \sin ^{2}[\theta(s)]\right\} .
$$

The functions $\alpha_{x}$ and $\alpha_{y}$ are related to the variables $\alpha_{x}$ and $\alpha_{y}$ in the cold-fluid equilibrium theory [6]. Indeed, by adding Eqs. (10) and (11) and subtracting Eq. (10) from Eq. (11) in [6], and carrying out the integrations on resulting equations with the initial condition $\alpha_{x}(0)=\alpha_{y}(0)=$ 0 , it is readily shown that the functions $\alpha_{x}$ and $\alpha_{y}$ in Eqs. (13) and (14) correspond to a particular solution to Eqs. (10) and (11) in [6].

As a second step, we apply another transformation from the twisted canonical variables $\left(\tilde{x}, \tilde{y}, \tilde{P}_{x}, \tilde{P}_{y}\right)$ to the canonical variables $\left(x_{1}, y_{1}, P_{x 1}, P_{y 1}\right)$ using the generating function

$$
\begin{aligned}
\tilde{F}_{2}\left(\tilde{x}, \tilde{y} ; P_{x 1}, P_{y 1}, s\right)= & \frac{1}{2}\left[\frac{w_{x}^{\prime}(s)}{w_{x}(s)}-C(s)\right] \tilde{x}^{2} \\
& +\frac{1}{2}\left[\frac{w_{y}^{\prime}(s)}{w_{y}(s)}+C(s)\right] \tilde{y}^{2} \\
& +\frac{\tilde{x} P_{x 1}}{w_{x}(s)}+\frac{\tilde{y} P_{y 1}}{w_{y}(s)},
\end{aligned}
$$

where $w_{x}(s)=w_{x}(s+S)$ and $w_{y}(s)=w_{y}(s+S)$ are periodic functions solving the differential equations

$$
\begin{aligned}
& \frac{w_{x}^{\prime \prime}(s)}{w_{x}(s)}-C^{\prime}(s)-C^{2}(s)-\alpha_{y}-\frac{2 K}{a(s)[a(s)+b(s)]} \\
& \quad=\frac{1}{w_{x}^{4}(s)}, \\
& \frac{w_{y}^{\prime \prime}(s)}{w_{y}(s)}+C^{\prime}(s)-C^{2}(s)-\alpha_{x}-\frac{2 K}{b(s)[a(s)+b(s)]} \\
& \quad=\frac{1}{w_{y}^{4}(s)} .
\end{aligned}
$$

It follows from Eq. (15) that

$$
\begin{aligned}
\tilde{P}_{x} & =\left[\frac{w_{x}^{\prime}(s)}{w_{x}(s)}-C(s)\right] \tilde{x}+\frac{P_{x 1}}{w_{x}(s)}, \\
\tilde{P}_{y} & =\left[\frac{w_{y}^{\prime}(s)}{w_{y}(s)}+C(s)\right] \tilde{y}+\frac{P_{y 1}}{w_{y}(s)}, \\
x_{1} & =\frac{\tilde{x}}{w_{x}(s)}, \\
y_{1} & =\frac{\tilde{y}}{w_{y}(s)} .
\end{aligned}
$$

The Hamiltonian in the canonical coordinate $\left(x_{1}, y_{1}, P_{x 1}, P_{y 1}\right)$ is then expressed as

$$
\begin{aligned}
H_{1 \perp}\left(x_{1}, y_{1}, P_{x 1}, P_{y 1}, s\right)= & \tilde{H}_{\perp}\left(\tilde{x}, \tilde{y}, \tilde{P}_{x}, \tilde{P}_{y}, s\right)+\frac{\partial \tilde{F}_{2}}{\partial s} \\
= & \frac{1}{2}\left\{\frac{P_{x 1}^{2}}{w_{x}^{2}(s)}+\frac{P_{y 1}^{2}}{w_{y}^{2}(s)}+\frac{x_{1}^{2}}{w_{x}^{2}(s)}\right. \\
& \left.+\frac{y_{1}^{2}}{w_{y}^{2}(s)}\right\} \\
& +\frac{d \varphi(s)}{d s}\left(y_{1} P_{x 1}-x_{1} P_{y 1}\right),
\end{aligned}
$$

where we have introduced and demanded

$$
\frac{d \varphi(s)}{d s} \equiv \frac{w_{y}(s)}{w_{x}(s)}\left\{\frac{d \theta(s)}{d s}-\alpha_{x}\right\}=\frac{w_{x}(s)}{w_{y}(s)}\left\{\frac{d \theta(s)}{d s}-\alpha_{y}\right\} .
$$

Following Eq. (20), it can be shown that the twisted angle $\theta(s)$ has to satisfy the differential equation

$$
\frac{d \theta(s)}{d s}=\frac{w_{x}^{2}(s) \alpha_{y}(s)-w_{y}^{2}(s) \alpha_{x}(s)}{w_{x}^{2}(s)-w_{y}^{2}(s)} .
$$

The motion described by the simplified Hamiltonian in the new canonical coordinate $\left(x_{1}, y_{1}, P_{x 1}, P_{y 1}\right)$ in Eq. (19) is described by the equations

$$
\begin{aligned}
x_{1}^{\prime} & =\frac{\partial H_{1 \perp}}{\partial P_{x 1}}=\frac{P_{x 1}}{w_{x}^{2}(s)}+\frac{d \varphi(s)}{d s} y_{1}, \\
y_{1}^{\prime} & =\frac{\partial H_{1 \perp}}{\partial P_{y 1}}=\frac{P_{y 1}}{w_{y}^{2}(s)}-\frac{d \varphi(s)}{d s} x_{1}, \\
P_{x 1}^{\prime} & =-\frac{\partial H_{1 \perp}}{\partial x_{1}}=-\frac{x_{1}}{w_{x}^{2}(s)}+\frac{d \varphi(s)}{d s} P_{y 1}, \\
P_{y 1}^{\prime} & =-\frac{\partial H_{1 \perp}}{\partial y_{1}}=-\frac{y_{1}}{w_{y}^{2}(s)}-\frac{d \varphi(s)}{d s} P_{x 1} .
\end{aligned}
$$

From Eq. (22), it is readily shown that

$$
\mathrm{E}=x_{1}^{2}+y_{1}^{2}+P_{x 1}^{2}+P_{y 1}^{2}
$$

is an exact single-particle constant of the motion for the Hamiltonian in Eq. (19).

We consider the following trial choice of the Vlasov equilibrium distribution function:

$f_{b}\left(x_{1}, y_{1}, P_{x 1}, P_{y 1}, s\right)=\frac{N_{b}}{\pi^{2} \varepsilon_{T}} \delta\left(x_{1}^{2}+y_{1}^{2}+P_{x 1}^{2}+P_{y 1}^{2}-\varepsilon_{T}\right)$,

where $d f_{b} / d s=0, \varepsilon_{T}=$ const $>0$ is an effective emittance, and $\delta(x)$ is the Dirac $\delta$ function. As will be shown in Sec. III, the density profile of the beam described by the distribution function $f_{b}$ is consistent with the uniformdensity profile within an ellipse, which is the key requirement for the quantity $\mathrm{E}=x_{1}^{2}+y_{1}^{2}+P_{x 1}^{2}+P_{y 1}^{2}$ to be a constant of motion. Therefore, the distribution function defined in Eq. (24) is indeed a Vlasov equilibrium, i.e., 


$$
\frac{\partial f_{b}}{\partial s}=0
$$

\section{STATISTICAL PROPERTIES}

The distribution function described in Eq. (24) has the following statistical properties. First, the distribution is consistent with the assumed density profile in Eq. (3), i.e.,

$$
\begin{aligned}
n_{b}(\tilde{x}, \tilde{y}, s) & =\frac{1}{w_{x} w_{y}} \iint f d P_{x 1} d P_{y 1} \\
& = \begin{cases}N_{b} /\left(\pi \varepsilon_{T} w_{x} w_{y}\right), & \text { if } x_{1}^{2} / \varepsilon_{T}+y_{1}^{2} / \varepsilon_{T} \leq 1, \\
0, & \text { otherwise. }\end{cases}
\end{aligned}
$$

The beam has the uniform-density profile given in Eq. (3), provided that $a=\sqrt{\varepsilon_{T}} w_{x}$ and $b=\sqrt{\varepsilon_{T}} w_{x}$ are satisfied. Under these self-consistent conditions, Eqs. (16), (17), and (21) can be expressed as

$$
\begin{aligned}
& \frac{d^{2} a}{d s^{2}}-\left[\frac{b^{2}\left(\alpha_{x}^{2}-2 \alpha_{x} \alpha_{y}\right)+a^{2} \alpha_{y}^{2}}{a^{2}-b^{2}}\right. \\
& \left.+\sqrt{\kappa_{z 0}} \frac{k_{0 x}^{2}-k_{0 y}^{2}}{k_{0}} \cos \left(k_{0} s\right) \sin (2 \theta)-2 \sqrt{\kappa_{z 0}} \alpha_{y} \sin \left(k_{0} s\right)\right] a \\
& -\frac{2 K}{a+b}=\frac{\varepsilon_{T}^{2}}{a^{3}},
\end{aligned}
$$$$
\frac{d^{2} b}{d s^{2}}+\left[\frac{a^{2}\left(\alpha_{y}^{2}-2 \alpha_{x} \alpha_{y}\right)+b^{2} \alpha_{x}^{2}}{a^{2}-b^{2}}\right.
$$$$
\left.+\sqrt{\kappa_{z 0}} \frac{k_{0 x}^{2}-k_{0 y}^{2}}{k_{0}} \cos \left(k_{0} s\right) \sin (2 \theta)+2 \sqrt{\kappa_{z 0}} \alpha_{x} \sin \left(k_{0} s\right)\right] b
$$$$
-\frac{2 K}{a+b}=\frac{\varepsilon_{T}^{2}}{b^{3}}
$$

$$
\frac{d \theta}{d s}=\frac{a^{2} \alpha_{y}-b^{2} \alpha_{x}}{a^{2}-b^{2}}
$$

Equations (27)-(29) are written in a form similar to the generalized envelope equations in the cold-fluid equilibrium theory [6]. They are identical to the generalized envelope equations of $a(s), b(s)$, and $\theta(s)$ in the cold-fluid equilibrium theory, except that the emittance terms appearing on the right-hand side of Eqs. (27) and (28) are zero in the cold-fluid equilibrium theory. Therefore, they are more general than the cold-fluid equilibrium theory.

Second, in the normalized units, the average (macroscopic flow) transverse velocity of the beam equilibrium described by Eq. (24) is given in the twisted coordinates by

$$
\begin{aligned}
\mathbf{V}_{\perp} & =\left(\frac{N_{b}}{\pi \varepsilon_{T} w_{x} w_{y}}\right)^{-1} \frac{1}{w_{x} w_{y}} \int \mathbf{v}_{\perp} f d P_{x 1} d P_{y 1} \\
& =\left(\frac{w_{x}^{\prime}}{w_{x}} \tilde{x}-\alpha_{x} \tilde{y}\right) \mathbf{e}_{\tilde{x}}+\left(\frac{w_{y}^{\prime}}{w_{y}} \tilde{y}+\alpha_{y} \tilde{x}\right) \mathbf{e}_{\tilde{y}} .
\end{aligned}
$$

The flow velocity in Eq. (30) is identical to the flow velocity derived by the cold-fluid equilibrium theory [6] provided that the relations $\mu_{x}=a^{\prime} / a=w_{x}^{\prime} / w_{x}$ and $\mu_{y}=$ $b^{\prime} / b=w_{y}^{\prime} / w_{y}$ are satisfied.

As a third statistical property, the beam equilibrium described by Eq. (24) has the effective transverse temperature profile (in dimensional units)

$$
\begin{aligned}
T_{\perp}(\tilde{x}, \tilde{y}, s) & =\left(\frac{N_{b}}{\pi \varepsilon_{T} w_{x} w_{y}}\right)^{-1} \frac{m \gamma_{b}}{2} \int\left(\mathbf{v}_{\perp}-\mathbf{V}_{\perp}\right)^{2} f d P_{x 1} d P_{y 1} \\
& =\frac{m c^{2} \gamma_{b} \beta_{b}^{2} \varepsilon_{T}^{2}}{2}\left(\frac{1}{a^{2}}+\frac{1}{b^{2}}\right)\left(1-\frac{\tilde{x}^{2}}{a^{2}}-\frac{\tilde{y}^{2}}{b^{2}}\right)
\end{aligned}
$$

As the fourth property, the rms emittances of the beam in the $\tilde{x}$ and the $\tilde{y}$ directions are

$$
\begin{aligned}
& \varepsilon_{t h \tilde{x}}=\frac{1}{\beta_{c} c} \sqrt{\left\langle\tilde{x}^{2}\right\rangle\left\langle\left(v_{\tilde{x}}-V_{\tilde{x}}\right)^{2}\right\rangle}=\frac{\varepsilon_{T}}{4}, \\
& \varepsilon_{t h \tilde{y}}=\frac{1}{\beta_{c} c} \sqrt{\left\langle\tilde{y}^{2}\right\rangle\left\langle\left(v_{\tilde{y}}-V_{\tilde{y}}\right)^{2}\right\rangle}=\frac{\varepsilon_{T}}{4} .
\end{aligned}
$$

Finally, the Vlasov elliptic beam equilibrium has two limiting cases which are well known. It recovers the familiar periodic (circular) rigid-rotor Vlasov equilibrium [10] by setting the major-axis equal to the minor axis of the beam ellipse. It also recovers the familiar constant-radius, uniform-density rigid-rotor Valsov equilibrium [9] by taking the limit of a uniform magnetic field with $B_{z}=B=$ const.

\section{EXAMPLES}

We illustrate examples of periodically twisted Vlasov elliptic beam equilibria in a periodic nonaxisymmetric magnetic focusing field and the temperature effects with numerical calculations. A numerical module in the PFB2D code $[6,21]$ has been developed to solve the generalized envelope equations (13), (14), and (27)-(29), which determines the rotational flow velocity, the outer equilibrium major axis $a(s)$ and minor axis $b(s)$ of the beam ellipse, and the twisted angle $\theta(s)$.

In particular, we consider a nonrelativistic elliptic beam with voltage $V_{b}=2.29 \mathrm{keV}$, current $I_{b}=0.11 \mathrm{~A}$, aspect ratio $a / b=6$, and nonaxisymmetric periodic permanent magnet focusing with $B_{0}=337.5 \mathrm{G}, S=1.912 \mathrm{~cm}$, and $k_{0 y} / k_{0 x}=1.6$, which is corresponding to a beam design for a high-efficiency $200 \mathrm{~W}$ RBA under development at Massachusetts Institute of Technology (MIT) and beam power technology for wireless communication. For such a system the matched solution of the generalized envelope equations (13), (14), and (27)-(29) is calculated numerically as shown in Figs. 2 and 3 for several maximum (onaxis) temperature choices and fixed parameters: $k_{0 x}=$ $1.90 \mathrm{~cm}^{-1}, \quad k_{0 y}=3.03 \mathrm{~cm}^{-1}, \quad \sqrt{\kappa_{z 0}}=1.04 \mathrm{~cm}^{-1}$, and $K=1.52 \times 10^{-2}$. The solutions to the generalized enve- 

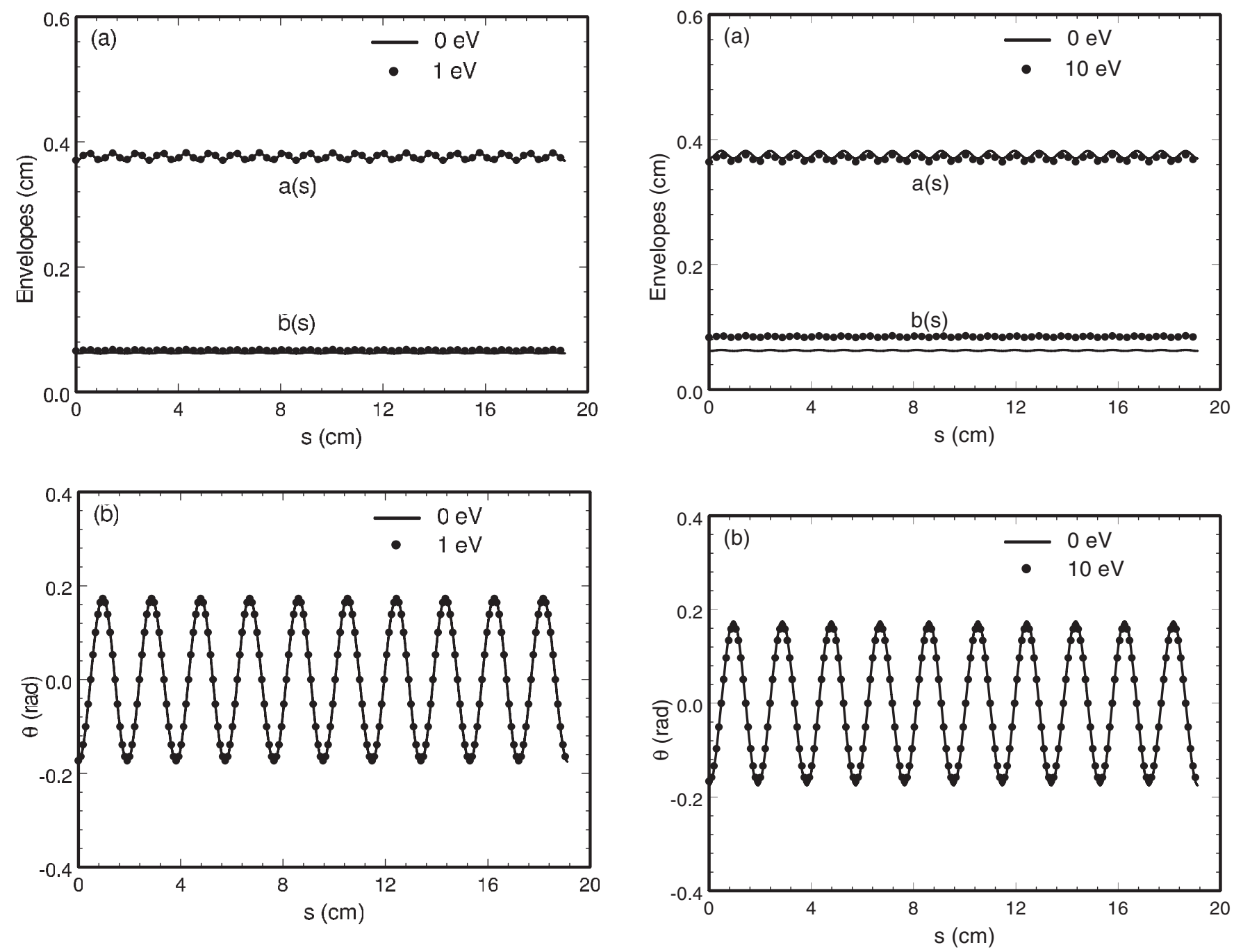

FIG. 2. Plots of (a) envelopes $a(s)$ and $b(s)$ and (b) twist angle $\theta(s)$ versus the axial distance $s$ for the nonrelativistic twisted elliptic beam. The solid curves are the generalized envelope solution for a zero-temperature beam, whereas the dotted curves are for a $1 \mathrm{eV}$ on-axis temperature beam.

lope equations (13), (14), and (27)-(29), displayed as solid and dotted curves in Figs. 2 and 3, show that the semiaxes of the elliptical beam remain almost constant with smallamplitude oscillations, that the orientation of the ellipse twists periodically with an amplitude of ten degrees.

The solid lines in Figs. 2 and 3 represent the beam envelopes and twisted angle with zero temperature which is corresponding to a cold beam, while the dotted curves represent the beam envelopes and twisted angles with $1 \mathrm{eV}$ on-axis temperature in Fig. 2 and $10 \mathrm{eV}$ on-axis temperature in Fig. 3, respectively. The aspect ratio of the beam reduces from 6 to 4 as the on-axis temperature of the beam increases from 0 to $10 \mathrm{eV}$, i.e., the elliptic beam becomes more circular. However, the twisted angle is almost unchanged as the on-axis temperature increases from 0 to $10 \mathrm{eV}$. For the elliptic beam designed for the $200 \mathrm{~W}$ ribbonbeam amplifier, the temperature of the beam is estimated to

FIG. 3. Plots of (a) envelopes $a(s)$ and $b(s)$ and (b) twist angle $\theta(s)$ versus the axial distance $s$ for the nonrelativistic twisted elliptic beam. The solid curves are the generalized envelope solution for a zero-temperature beam, whereas the dotted curves are for a $10 \mathrm{eV}$ on-axis temperature beam.

be $0.1 \mathrm{eV}$ from simulations $[22,23]$. In such a case, the temperature effect is negligible.

To further illustrate the effects of beam temperature, we consider a relativistic elliptic beam with $V_{b}=198.5 \mathrm{keV}$, current $I_{b}=85.5 \mathrm{~A}$, aspect ratio $a / b=5$, and nonaxisymmetric periodic permanent magnet focusing with $B_{0}=$ $2.4 \mathrm{kG}, S=2.2 \mathrm{~cm}$, and $k_{0 y} / k_{0 x}=1.52$. [Such a relativistic elliptic beam could be used in a $10 \mathrm{MW} L$-band RBK for the International Linear Collider (ILC).] For such a system the matched solution of the generalized envelope equations (13), (14), and (27)-(29) is calculated numerically with the corresponding parameters: $k_{0 x}=$ $1.57 \mathrm{~cm}^{-1}, \sqrt{\kappa_{z 0}}=0.732 \mathrm{~cm}^{-1}$, and $K=1.13 \times 10^{-2}$. As shown in Fig. 4, the solid lines represent the beam semiaxis envelopes and twist angle with zero temperature which is corresponding to a cold beam, while the dotted 

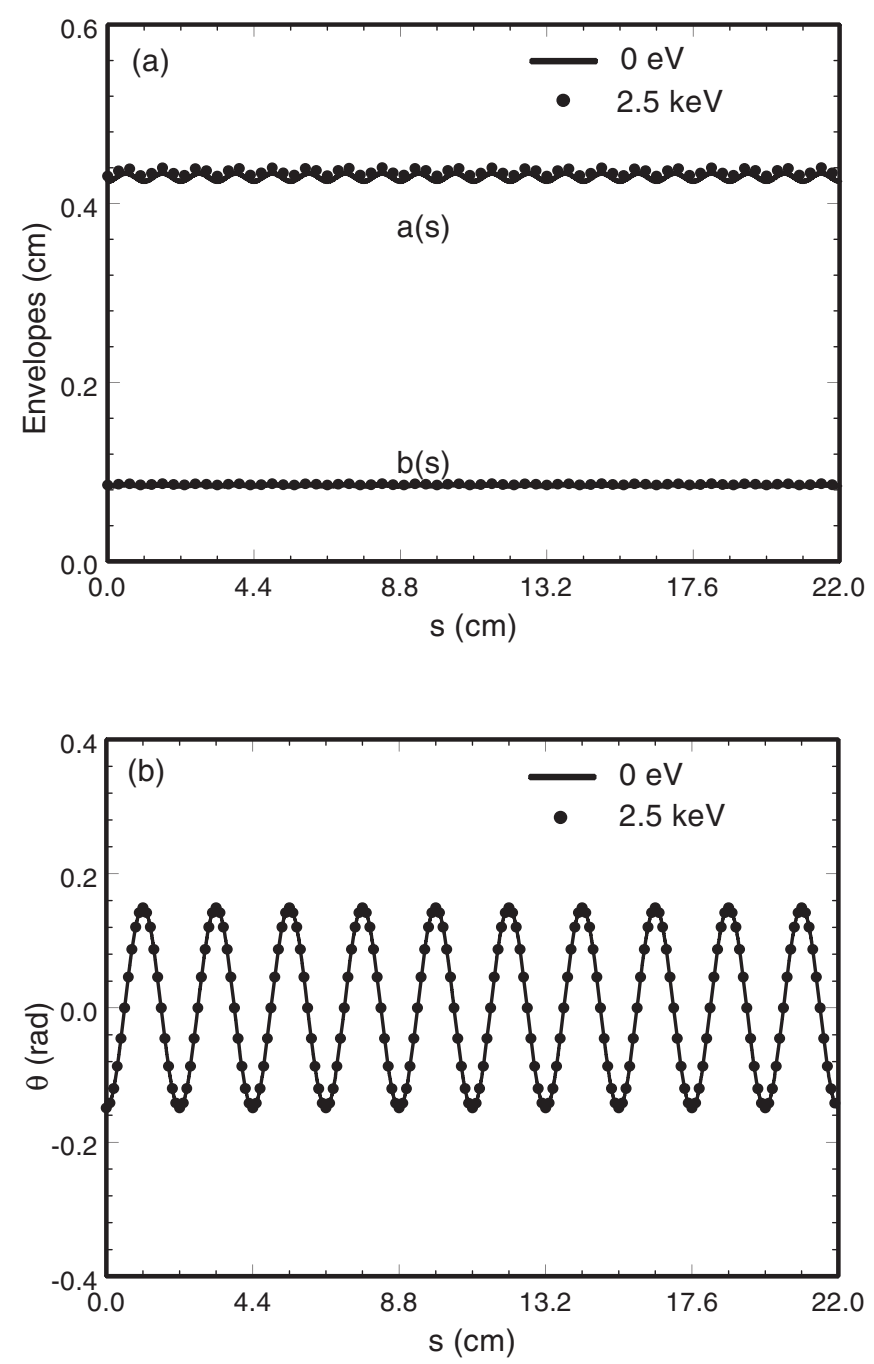

FIG. 4. Plots of (a) envelopes $a(s)$ and $b(s)$ and (b) twist angle $\theta(s)$ versus the axial distance $s$ for the relativistic twisted elliptic beam. The solid curves are the generalized envelope solution for a zero-temperature beam, whereas the dotted curves are for a $2.5 \mathrm{keV}$ on-axis temperature beam.

curves represent the beam envelopes and twist angle with $2.5 \mathrm{keV}$ on-axis temperature. It is evident in Fig. 4 that the temperature effects on the beam envelopes and twist angle are negligibly small. Since an actual relativistic elliptic beam in a well designed system will have a temperature which will be considerably less than $2.5 \mathrm{keV}$, the results in Fig. 4 imply that the temperature effect on the beam envelopes and twist angle is expected to be negligibly small.

\section{CONCLUSIONS}

The single-particle Hamiltonian of a periodically twisted large-aspect-ratio elliptic beam in a nonaxisymmetric periodic magnetic focusing field has been investigated. A constant of motion analogous to the CourantSnyder invariant has been found such that the self- consistent beam equilibrium can be constructed as a function of the constant of motion. The beam envelope equations and flow velocity equations have been derived. They are consistent with the generalized envelope equations derived from the cold-fluid equilibrium theory [6] when the temperature is taken to be zero. Statistical properties of the present Vlasov elliptic beam equilibrium have been studied. For current applications of interest, namely, the RBA and RBK, the temperature effects have been found to be negligibly small on periodically twisted large-aspectratio elliptic beams.

\section{ACKNOWLEDGMENTS}

This research was supported by the U.S. Department of Energy, Office of High-Energy Physics, Grant No. DEFG02-95ER40919, Office of Fusion Energy Science, Grant No. DE-FG02-01ER54662, and in part by Air Force Office of Scientific Research, Grant No. FA955006-1-0269.

[1] R. C. Davidson and C. Chen, Part. Accel. 59, 175 (1998); Nucl. Instrum. Methods Phys. Res., Sect. A 415, 370 (1998).

[2] R. C. Davidson, P. Stoltz, and C. Chen, Phys. Plasmas 4, 3710 (1997).

[3] R. Pakter and C. Chen, Phys. Rev. E 62, 2789 (2000).

[4] C. Chen and R. Pakter, Phys. Plasmas 7, 2203 (2000).

[5] S. Bernal et al., Proceedings of the 2005 Particle Accelerator Conference, 2005, p. 892.

[6] J. Zhou, R. Bhatt, and C. Chen, Phys. Rev. ST Accel. Beams 9, 034401 (2006).

[7] I. M. Kapchinskij and V. V. Vladimirskij, in Proceedings of the International Conference on High Energy Accelerators (CERN, Geneva, 1959), p. 274.

[8] S. M. Lund and B. Bukh, Phys. Rev. ST Accel. Beams 7, 024801 (2004).

[9] R. C. Davidson, Physics of Nonneutral Plasma (AddisonWesley, Reading, MA, 1990).

[10] C. Chen, R. Pakter, and R. C. Davidson, Phys. Rev. Lett. 79, 225 (1997).

[11] R. C. Davidson and H. Qin, Physics of Intense Charged Particle Beams in High Energy Accelerators (World Scientific, Singapore, 2001), p. 242.

[12] F. J. Sacherer, Ph.D. thesis, Lawrence Radiation Laboratory, University of California, Berkeley, 1968, pp. 15-19.

[13] V. Danilov, S. Cousineau, S. Henderson, and J. Holmes, Phys. Rev. ST Accel. Beams 6, 094202 (2003).

[14] C. Chen, R. Pakter, and R. C. Davidson, Nucl. Instrum. Methods Phys. Res., Sect. A 464, 518 (2001).

[15] P. A. Sturrock, J. Electron. Control 7, 162 (1959).

[16] Z.X. Zhang et al., IEEE Trans. Plasma Sci. 21, 760 (1993).

[17] J.H. Booske, A.H. Kumbasar, and M. A. Basten, Phys. Rev. Lett. 71, 3979 (1993). 
[18] M. A. Basten and J.H. Booske, J. Appl. Phys. 85, 6313 (1999).

[19] S. J. Russell et al., Phys. Rev. ST Accel. Beams 8, 080401 (2005).

[20] E. D. Courant and H. S. Snyder, Ann. Phys. (N.Y.) 3, 1 (1958).
[21] J. Zhou, Ph.D. thesis, Massachusetts Institute of Technology, 2006.

[22] R. Bhatt and C. Chen, Phys. Rev. ST Accel. Beams 8, 014201 (2005).

[23] R. Bhatt, T. Bemis, and C. Chen, IEEE Trans. Plasma Sci. 34, 187 (2006). 